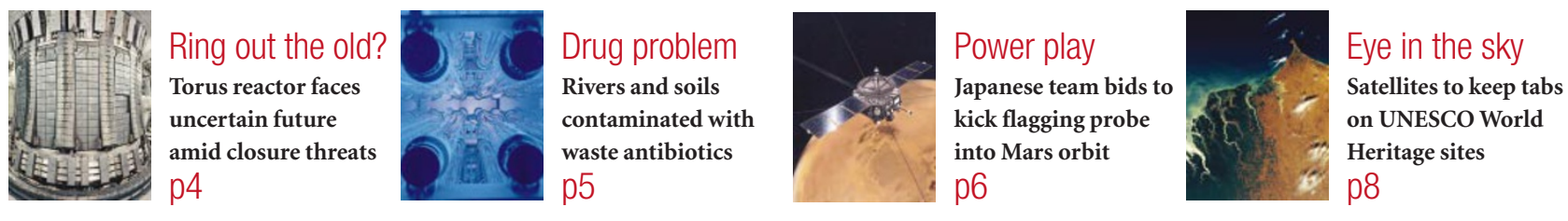

\title{
Below-par performance hampers Fermilab quest for Higgs boson
}

\section{Geoff Brumfiel, Washington}

Physicists at the Tevatron particle accelerator near Chicago are steeling themselves for failure in their ambitious bid to detect the elusive Higgs boson.

Researchers working on the machine, at the Fermi National Accelerator Laboratory (Fermilab) in Batavia, Illinois, are searching for signs of the particle, thought to give other particles their mass, in the debris of highenergy particle collisions.

But they have now cut their estimate of the number of proton-antiproton collisions they expect to see by 2008 by $60-80 \%$. As many collisions have to be studied to detect the Higgs boson, this is a serious blow to the lab's hopes of observing the particle.

The figures appear in a document prepared for Fermilab's sponsor, the US Department of Energy, and released on 15 June. Stephen Holmes, associate director for accelerators at the lab, says that problems have arisen with the equipment used to accelerate the protons and antiprotons. The Tevatron is 20 years old and its accelerators have been plagued by trouble during 'Run II', an upgraded second phase of operation that began in 2001.

The news is forcing Michael Witherell, Fermilab's director, to reconsider funding for Run II, which consumes nearly two-thirds of the lab's \$300-million annual budget. "We have to make some tough choices," he says.

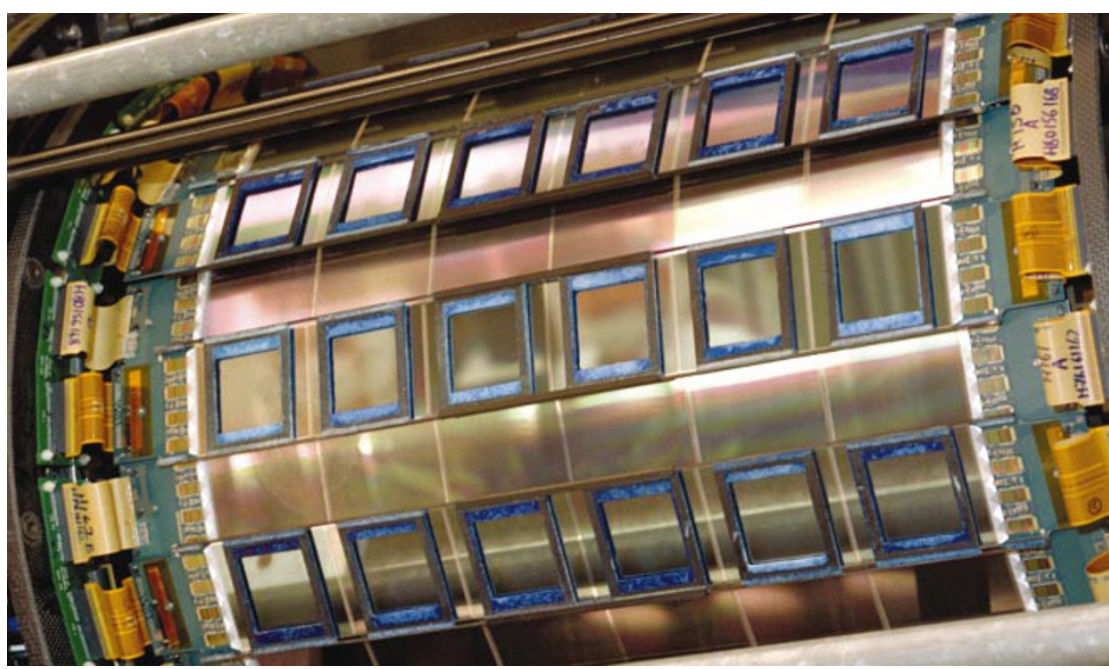

Poor results mean that Fermilab may not splash out on new silicon wafers for its particle detectors.

Witherell says he may have to withhold $\$ 25$ million needed to replace the detectors' silicon wafers, which create electrical signals when hit by particles. Researchers warn that this could severely impair the detectors' performance.

Many observers doubt whether the Tevatron will be able to find the Higgs boson before the rival Large Hadron Collider comes online in 2007 at CERN, the European particle-physics lab near Geneva. Witherell puts the chance of spotting the Higgs at "something like 50\%". Others disagree: "I don't think there's any chance they will find it," says CERN physicist Daniel Froidevaux.

Many Fermilab researchers admit that they placed too much emphasis on finding the Higgs. Now, they say, they need to draw attention to their other research, such as studies of the top quark, a subatomic particle that was discovered at the lab in 1995. "I think we need to get some buttons out there that say: 'Run II, it ain't just the Higgs',' says Holmes.

\section{String theorists bypass NSF en route to Iran seminar}

\section{Geoff Brumfiel, Washington}

Despite increasing political tension, a group of US researchers are standing by their pledge to visit Iran this autumn for a workshop on superstrings.

Lack of money almost forced the team to abandon the trip. Getting the funds has been difficult, says trip organizer Albion Lawrence of Brandeis University in Waltham,

Massachusetts. Fundraising was delayed for months by the war in Iraq and the current row over Iran's nuclear programme.

But on 25 June the problem was solved for seven members of the twelve-strong group. The Clay Mathematics Institute, a Bostonbased charity, pledged $\$ 12,000$ for the researchers to travel to Tehran. The National Science Foundation (NSF) has denied funds to graduate students who want to attend.

The meeting is organized by the Iranian Institute for Studies in Theoretical Physics and Mathematics in Tehran, which has held an annual school and workshop on string theory for the past three years.

This year's conference, near Anzali, on the Caspian Sea, from 29 September to
9 October, will be the first to have significant US participation. "This is an important development in scientific relations between US and Iranian scientists," says Farhad Ardalan, a theorist at the Tehran institute and one of the meeting's organizers.

String theory postulates that particles can be described as vibrating loops or strings. "In the past few years, a strong group of string theorists has developed in Iran," says Eva Silverstein, a theorist at the Stanford Linear Accelerator Center in California who will attend the meeting. 